\title{
Magnetized static black Saturn
}

\author{
Stoytcho S. Yazadjiev * \\ Institut für Theoretische Physik, Universität Göttingen, \\ Friedrich-Hund-Platz 1, D-37077 Göttingen, Germany \\ and \\ Department of Theoretical Physics, Faculty of Physics, Sofia University, \\ 5 James Bourchier Boulevard, Sofia 1164, Bulgaria
}

\begin{abstract}
We construct a new static solution to the 5D Einstein-Maxwell equations describing a static black hole surrounded by a non-rotating dipole black ring. The configuration is kept in equilibrium by an external magnetic field interacting with the dipole charge of the black ring. The properties of the black Saturn-like configuration are studied and the basic physical quantities are calculated. The solution demonstrates 2-fold continuous non-uniqueness of the 5D magnetized static neutral black objects for fixed total mass and Melvin background.
\end{abstract}

\section{Introduction}

In the last years we observe rapidly growing interest in the higher dimensional black holes. Only in five dimensions, however, we have exact solutions describing more nontrivial black objects as black rings, black Saturns, concentric and orthogonal black di-rings [1]-[13]. Some basic steps towards the classification of the five dimensional asymptotically flat black solutions were also made [14, [15]. Nevertheless, our knowledge of the 5D black objects and their physical properties is rather incomplete. Let us mention some of the open problems. Are there black holes and black rings with rod structure different from the standard one? Are there black Saturns and black multirings for which the balancing mechanism is different, or partially different from the centrifugal repulsion? In particular, are there static charged black Saturns and multirings where the electric charge balances the gravitational attraction? In this situation the exact solutions remain almost the only way to obtain new valuable and reliable knowledge of the black objects and their physical properties. Most of the known exact solutions describing black objects with nontrivial horizon topology are asymptotically flat. There are, however, asymptotically non-flat black solutions which are also interesting from physical point of view. As an example we can mention the so-called

*E-mail: yazadj@theorie.physik.uni-goettingen.de; yazad@phys.uni-sofia.bg 
magnetized solutions describing various kinds of objects immersed in a self-gravitating magnetic field [16], [17].

The aim of this paper is to present a new static solution describing a non-rotating black hole surrounded by a non-rotating dipole black ring in the 5D Einstein-Maxwell (EM) gravity. The described solution will be called magnetized, static, dipole black Saturn. In contrast to the case of the known black Saturn and di-ring solutions [9][13] where the black configurations are balanced by the rotation of the black ring(s), in our case the balancing mechanism is quite different. The configuration is kept in equilibrium by a self-gravitating external magnetic field interacting with the dipole charge. This interaction, namely, provides the additional force which can balance the configuration.

\section{Static dipole black Saturn in external magnetic field}

The 5D EM gravity is described by the field equations

$$
\begin{aligned}
& R_{\mu \nu}=\frac{1}{2}\left(F_{\mu \lambda} F_{\nu}^{\lambda}-\frac{1}{6} F_{\sigma \lambda} F^{\sigma \lambda} g_{\mu \nu}\right), \\
& \nabla_{\mu} F^{\mu \nu}=0=\nabla_{[\mu} F_{\nu \sigma]} .
\end{aligned}
$$

One of the ways to construct the magnetized static dipole black Saturn solution to the field equations (11) is to magnetize first the stationary dipole black Saturn of [10] and then take the static limit. The disadvantage of this approach is the fact that the explicit form of the gauge potential is not known for the stationary dipole black Saturn. That is why, we prefer to use more constructive approach in order to present the solution in completely explicit form. Namely, using the solution generating method of [14] we generate first the configuration 1 of a static black hole surrounded by a static dipole black ring. Then we magnetize this static solution by applying the Harrison transformation [16], [17]. As a result of the procedure described we obtain the following explicit solution

$$
\begin{aligned}
& d s^{2}=\frac{\Lambda}{S}\left[\mathcal{G}_{t} d t^{2}+Y^{3} e^{2 K}\left(d \rho^{2}+d z^{2}\right)+\mathcal{G}_{\psi} d \psi^{2}\right]+\frac{S^{2}}{\Lambda^{2}} \mathcal{G}_{\phi} d \phi^{2} \\
& A_{\phi}=\Lambda^{-1}\left[\mathcal{U}_{\phi}+\frac{\sqrt{3} b}{2}\left(S^{2} \mathcal{G}_{\phi}+\frac{4}{3} \mathcal{U}_{\phi}^{2}\right)\right]
\end{aligned}
$$

where

$$
\Lambda=\frac{1}{3} B^{2} S^{2} \mathcal{G}_{\phi}+\left(1+\frac{2}{3} B \mathcal{U}_{\phi}\right)^{2}
$$

and the functions $\mathcal{G}_{t}, \mathcal{G}_{\psi}, \mathcal{G}_{\phi}, e^{2 K}, S, Y$, and $\mathcal{U}_{\phi}$ are given by

$$
\mathcal{G}_{t}=-\frac{\mu_{1} \mu_{3}}{\mu_{2} \mu_{4}}, \quad \mathcal{G}_{\psi}=\mu_{2}, \quad \mathcal{G}_{\phi}=\frac{\rho^{2} \mu_{4}}{\mu_{1} \mu_{3}},
$$

\footnotetext{
${ }^{1}$ Although it is clear it is worth noting that this configuration suffers from the presence of conical singularities.
} 


$$
\begin{aligned}
& e^{2 K}=e^{2 K_{0}} \frac{\mu_{2}\left(\rho^{2}+\mu_{1} \mu_{2}\right)\left(\rho^{2}+\mu_{1} \mu_{4}\right)^{2}\left(\rho^{2}+\mu_{2} \mu_{3}\right)\left(\rho^{2}+\mu_{3} \mu_{4}\right)^{2}}{\left(\rho^{2}+\mu_{1} \mu_{3}\right)^{2}\left(\rho^{2}+\mu_{2} \mu_{4}\right) \prod_{i=1}^{4}\left(\rho^{2}+\mu_{i}^{2}\right)} \\
& S=\frac{(1-\omega)(1-\nu) R_{1}+(1+\omega)(1+\nu) R_{4}+2(\omega+\nu) R_{3}+\omega\left(1-\nu^{2}\right) \mathcal{R}^{2}}{(1-\omega)(1-\nu) R_{1}+(1+\omega)(1+\nu) R_{4}+2(\omega+\nu) R_{3}-\omega\left(1-\nu^{2}\right) \mathcal{R}^{2}} \\
& Y=\frac{(1-\omega)(1-\nu) R_{1}+(1+\omega)(1+\nu) R_{4}+2(\omega+\nu) R_{3}+\omega\left(1-\nu^{2}\right) \mathcal{R}^{2}}{\left[(1-\nu) R_{1}+(1+\nu) R_{4}+2 \nu R_{3}\right]} \\
& \mathcal{U}_{\phi}=\frac{2 \sqrt{3}(1-\nu) \mathcal{R} C(\nu,-\omega)\left[R_{1}-R_{3}+\frac{1}{2}(1+\nu) \mathcal{R}^{2}\right]}{(1-\omega)(1-\nu) R_{1}+(1+\omega)(1+\nu) R_{4}+2(\nu+\omega) R_{3}-\omega\left(1-\nu^{2}\right) \mathcal{R}^{2}}
\end{aligned}
$$

The functions $R_{i}$ and $\mu_{i}$ are defined by

$$
R_{i}=\sqrt{\rho^{2}+\left(z-a_{i}\right)^{2}}, \quad \mu_{i}=R_{i}-\left(z-a_{i}\right) .
$$

In all the above expressions $a_{i}, \omega, \nu, \mathcal{R}$ and $B$ are real constants with

$$
a_{1}=-\frac{\nu}{2} \mathcal{R}^{2}, \quad a_{2}=\frac{\zeta}{2} \mathcal{R}^{2}, \quad a_{3}=\frac{1}{2} \mathcal{R}^{2}, \quad a_{4}=\frac{\nu}{2} \mathcal{R}^{2}, \quad C(\nu,-\omega)=\sqrt{\omega(\omega+\nu) \frac{1-\omega}{1+\omega}}
$$

where 2

$$
0 \leq \omega<1, \quad 0 \leq \nu<1, \quad 1 \leq \zeta
$$

The solution depends on six independent parameters $-\omega, \nu, \zeta, \mathcal{R}, B(B>0)$ and $e^{2 K_{0}}$. The parameter $B$, as we will see, is the asymptotic strength of the external magnetic field.

\section{Analysis of the solution}

\subsection{Asymptotic behavior}

In order to study the asymptotic behaviour of the solution we introduce the asymptotic coordinates $r$ and $\theta$ defined by

$$
\rho=\frac{1}{2} r^{2} \sin 2 \theta, \quad z=\frac{1}{2} r^{2} \cos 2 \theta
$$

Then in the asymptotic limit $r \rightarrow \infty$ we find

$$
\begin{aligned}
& \mathcal{G}_{t} \approx-1+(2 \nu+\zeta-1) \frac{\mathcal{R}^{2}}{r^{2}} \\
& \mathcal{G}_{\psi} \approx r^{2} \sin ^{2} \theta \\
& \mathcal{G}_{\phi} \approx r^{2} \cos ^{2} \theta \\
& S \approx 1+\frac{2 \omega(1-\nu)}{1+\omega} \frac{\mathcal{R}^{2}}{r^{2}}
\end{aligned}
$$

\footnotetext{
${ }^{2}$ In other words we have $a_{1} \leq a_{4}<a_{3} \leq a_{2}$.
} 


$$
\begin{aligned}
& Y \approx(1+\omega)\left[1+\frac{2 \omega(1-\nu)}{1+\omega} \frac{\mathcal{R}^{2}}{r^{2}} \sin ^{2} \theta\right], \\
& e^{2 K} \approx \frac{e^{2 K_{0}}}{r^{2}} \\
& \mathcal{U}_{\phi} \approx \frac{2 \sqrt{3}(1-\nu) \mathcal{R} C(\nu,-\omega)}{1+\omega} \frac{\mathcal{R}^{2}}{r^{2}} \cos ^{2} \theta .
\end{aligned}
$$

Therefore the asymptotic form of the solution is the following

$$
\begin{aligned}
d s^{2} \approx & \left(1+\frac{1}{3} B^{2} r^{2} \cos ^{2} \theta\right)\left[-d t^{2}+(1+\omega)^{3} e^{2 K_{0}}\left(d r^{2}+r^{2} d \theta^{2}\right)+r^{2} \sin ^{2} \theta d \psi^{2}\right] \\
& +\frac{r^{2} \cos ^{2} \theta d \phi^{2}}{\left(1+\frac{1}{3} B^{2} r^{2} \cos ^{2} \theta\right)^{2}}, \\
A_{\phi} \approx \quad & \frac{1}{2} B \frac{r^{2} \cos ^{2} \theta}{\left(1+\frac{1}{3} B^{2} r^{2} \cos ^{2} \theta\right)} .
\end{aligned}
$$

With the choice

$$
e^{2 K_{0}}=(1+\omega)^{-3}
$$

the solution approaches the 5D Melvin universe with external axial magnetic field $B$. From now on we adopt that the constant $e^{2 K_{0}}$ is given by (23).

\subsection{Rod structure}

The rod structure is the following.

(i) Semi-infinite rod $\left(-\infty, a_{1}\right]$ and the finite rod $\left[a_{4}, a_{3}\right]$ corresponding to the $\phi \phi$-part of the metric

In order to cure the conical singularities at the location of the semi-infinite rod the coordinate $\phi$ must have a period

$$
\Delta \phi=2 \pi \lim _{\rho \rightarrow 0} \sqrt{\frac{\rho^{2} g_{\rho \rho}}{g_{\phi \phi}}}
$$

which gives

$$
\Delta \phi=2 \pi(1+\omega)^{3 / 2} e^{K_{0}} \Lambda^{3 / 2}(\rho=0)=2 \pi .
$$

Respectively, for the finite rod $\left[a_{4}, a_{3}\right]$ we find

$$
\Delta \phi=2 \pi \lim _{\rho \rightarrow 0} \sqrt{\frac{\rho^{2} g_{\rho \rho}}{g_{\phi \phi}}}=2 \pi(1-\omega)^{3 / 2} e^{K_{0}}\left(\frac{1-\nu}{1+\nu}\right) \sqrt{\frac{\zeta+\nu}{\zeta-\nu}} \Lambda^{3 / 2}(\rho=0) .
$$

For the rod $\left[a_{4}, a_{3}\right]$ we have

$$
\Lambda(\rho=0)=\left(1+\frac{2}{3} B \mathcal{Q}\right)^{2}
$$


and therefore we can write

$$
\Delta \phi=2 \pi\left(\frac{1-\omega}{1+\omega}\right)^{3 / 2}\left(\frac{1-\nu}{1+\nu}\right) \sqrt{\frac{\zeta+\nu}{\zeta-\nu}}\left(1+\frac{2}{3} B \mathcal{Q}\right)^{3} .
$$

Here $\mathcal{Q}$ is the dipole charge of the non-magnetized dipole black ring. The explicit formula for $\mathcal{Q}$ is presented below (see eq. (53)).

(ii) Semi-infinite rod $\left[a_{2}, \infty\right)$ corresponding to the $\psi \psi$-part of the metric

The regularity condition here gives

$$
\Delta \psi=2 \pi \lim _{\rho \rightarrow 0} \sqrt{\frac{\rho^{2} g_{\rho \rho}}{g_{\psi \psi}}}=2 \pi(1+\omega)^{3 / 2} e^{K_{0}}=2 \pi .
$$

In order to find the balancing condition we must impose the r.h.s of (28) to be equal to that of (25). In other words the balancing condition is

$$
\left(\frac{1-\omega}{1+\omega}\right)^{3 / 2}\left(\frac{1-\nu}{1+\nu}\right) \sqrt{\frac{\zeta+\nu}{\zeta-\nu}}\left(1+\frac{2}{3} B \mathcal{Q}\right)^{3}=1 .
$$

The eq.(30) can be solved to determine $B$ as a function of the remaining parameters. In other words the external magnetic field can always be chosen such as to cancel the conical singularity and to support the static Saturn in equilibrium. This is possible because of the coupling between the external magnetic filed $B$ and the dipole charge $\mathcal{Q}$. This coupling provides the additional force which compensates the gravitational attraction between the black hole and the black ring and the magnetic self-interaction of the dipole black ring.

\subsection{Horizons}

\subsubsection{Black ring horizon}

The black ring horizon is located at $\rho=0$ for $a_{1} \leq z \leq a_{4}$. The metric of the spacial cross section of the black ring horizon is given by

$$
d s_{B R}^{2}=\frac{\Lambda_{B R}}{S_{B R}}\left[Y_{B R}^{3} e^{2 K_{B R}} d z^{2}+\mathcal{G}_{\psi}^{B R} d \psi^{2}\right]+\frac{S_{B R}^{2}}{\Lambda_{B R}^{2}} \mathcal{G}_{\phi}^{B R} d \phi^{2}
$$

where

$$
\begin{aligned}
S_{B R} & =\frac{\frac{1}{2} \mathcal{R}^{2}-z}{\frac{1}{2} \mathcal{R}^{2} \frac{\nu(1+\nu \omega)}{\omega+\nu}-z}, \\
Y_{B R} & =\frac{\omega+\nu}{\nu} \\
e^{2 K_{B R}} & =\mathcal{R}^{2} \frac{(\zeta+\nu) \nu^{2}}{(1+\nu)^{2}(1+\omega)^{3}} \frac{\left(\frac{1}{2} \mathcal{R}^{2}-z\right)}{\left(z+\frac{\nu}{2} \mathcal{R}^{2}\right)\left(\frac{\nu}{2} \mathcal{R}^{2}-z\right)\left(\frac{\zeta}{2} \mathcal{R}^{2}-z\right)} \\
\mathcal{G}_{\psi}^{B R} & =2\left(\frac{\zeta}{2} \mathcal{R}^{2}-z\right)
\end{aligned}
$$




$$
\begin{aligned}
\mathcal{G}_{\phi}^{B R} & =2 \frac{\left(z+\frac{\nu}{2} \mathcal{R}^{2}\right)\left(\frac{\nu}{2} \mathcal{R}^{2}-z\right)}{\left(\frac{1}{2} \mathcal{R}^{2}-z\right)}, \\
\Lambda_{B R} & =b^{2} S_{B R}^{2} \mathcal{G}_{\phi}^{B R}+\left(1+\frac{2 b}{3} \mathcal{U}_{\phi}^{B R}\right)^{2}, \\
\mathcal{U}_{\phi}^{B R} & =\frac{\sqrt{3}(1-\nu) \mathcal{R} C(\nu,-\omega)}{(\omega+\nu)} \frac{\left(z+\frac{\nu}{2} \mathcal{R}^{2}\right)}{\left(\frac{\nu(1+\omega \nu)}{2(\omega+\nu)} \mathcal{R}^{2}-z\right)} .
\end{aligned}
$$

Since the orbits of $\phi$ shrink to zero size at $z=a_{1}=-\frac{\nu}{2} \mathcal{R}^{2}$ and $z=a_{4}=\frac{\nu}{2} \mathcal{R}^{2}$ and the orbits of $\psi$ do not shrink to zero size anywhere (for $a_{1} \leq z \leq a_{4}$ ), the topology of the horizon is $S^{2} \times S^{1}$. Metrically, however, the horizon is distorted by the external magnetic field and the gravitational attraction by the black hole. The area of the horizon can be found by direct calculations

$$
\mathcal{A}_{B R}=8 \pi^{2} \frac{\mathcal{R}^{3}}{(1+\nu)} \sqrt{\nu(\zeta+\nu)\left(\frac{\omega+\nu}{1+\omega}\right)^{3}} .
$$

The temperature of the black ring horizon can be obtained form the surface gravity and it is given by

$$
T_{B R}=\frac{1+\nu}{4 \pi \mathcal{R}} \sqrt{\frac{\nu}{\zeta+\nu}\left(\frac{1+\omega}{\omega+\nu}\right)^{3}} .
$$

The same result is obtained by using the euclidian approach.

Let us note that the area and the temperature of the black ring horizon are not affected by the external magnetic field and they coincide with the corresponding quantities of the non-magnetized solution. This is in complete agreement with what was

observed for other the magnetized static black solutions - the external magnetic field does not affect the thermodynamics [19], [16], [17].

\subsubsection{Black hole horizon}

The black ring horizon is located at $\rho=0$ for $a_{3} \leq z \leq a_{2}$. The metric of the spacial cross section of the black hole horizon is

$$
d s_{B H}^{2}=\frac{\Lambda_{B H}}{S_{B H}}\left[Y_{B H}^{3} e^{2 K_{B H}} d z^{2}+\mathcal{G}_{\psi}^{B H} d \psi^{2}\right]+\frac{S_{B H}^{2}}{\Lambda_{B H}^{2}} \mathcal{G}_{\phi}^{B H} d \phi^{2}
$$

and the metric functions are given as follows

$$
\begin{aligned}
& S_{B H}=\frac{z-\frac{\nu}{2} \mathcal{R}^{2}}{z-\frac{2 \omega+\nu(1-\omega)}{2(1+\omega)} \mathcal{R}^{2}}, \\
& Y_{B H}=1+\omega \\
& e^{2 K_{B H}}=\mathcal{R}^{2} \frac{(\zeta+\nu)(\zeta-1)}{4(1+\omega)^{3}(\zeta-\nu)} \frac{\left(z-\frac{\nu}{2} \mathcal{R}^{2}\right)}{\left(z+\frac{\nu}{2} \mathcal{R}^{2}\right)\left(\frac{\zeta}{2} \mathcal{R}^{2}-z\right)\left(z-\frac{1}{2} \mathcal{R}^{2}\right)} \\
& \mathcal{G}_{\psi}^{B H}=2\left(\frac{\zeta}{2} \mathcal{R}^{2}-z\right)
\end{aligned}
$$




$$
\begin{aligned}
\mathcal{G}_{\phi}^{B H} & =2 \frac{\left(z+\frac{\nu}{2} \mathcal{R}^{2}\right)\left(z-\frac{1}{2} \mathcal{R}^{2}\right)}{\left(z-\frac{\nu}{2} \mathcal{R}^{2}\right)}, \\
\Lambda_{B H} & =b^{2} S_{B H}^{2} \mathcal{G}_{\phi}^{B H}+\left(1+\frac{2 b}{3} \mathcal{U}_{\phi}^{B H}\right)^{2}, \\
\mathcal{U}_{\phi}^{B H} & =\frac{\sqrt{3}(1-\nu) \mathcal{R}^{3} C(\nu,-\omega)}{(1+\omega)\left(z-\frac{2 \omega+\nu(1-\omega)}{2(1+\omega)} \mathcal{R}^{2}\right)} .
\end{aligned}
$$

The orbits of $\phi$ shrink to zero size at $z=a_{3}=\frac{1}{2} \mathcal{R}^{2}$ and the orbits of $\psi$ at $z=$ $a_{2}=\frac{\zeta}{2} \mathcal{R}^{2}$. Therefore the topology of the horizon is $S^{3}$. The 3 -sphere of the horizon is metrically distorted by the self-gravitating external magnetic field and the gravitational attraction of the black ring.

The area of the black hole horizon is

$$
\mathcal{A}_{B H}=2 \pi^{2} \mathcal{R}^{3} \sqrt{\frac{(\zeta+\nu)(\zeta-1)^{3}}{(\zeta-\nu)}}
$$

and temperature of the black hole is given by

$$
T_{B H}=\frac{1}{2 \pi \mathcal{R}} \sqrt{\frac{\zeta-\nu}{(\zeta+\nu)(\zeta-1)}} .
$$

The area and the temperature of the black hole are not affected by the external magnetic field.

\subsection{Mass and dipole charge}

The dipole charge is defined by

$$
\mathcal{Q}_{M}=\frac{1}{2 \pi} \oint_{S^{2}} F
$$

where $S^{2}$ is the two-dimensional sphere of the black ring horizon. The subscript "M" means that the dipole charge is for the magnetized solution. By straightforward calculations we find that the dipole charge is given by

$$
\mathcal{Q}_{M}=\frac{\mathcal{Q}}{1+\frac{2}{3} B \mathcal{Q}}
$$

where

$$
\mathcal{Q}=2 \sqrt{3} \mathcal{R} \frac{C(\nu,-\omega)}{1-\omega}
$$

is the dipole charge of the non-magnetized dipole black ring.

In order to compute the mass of our black configuration we use the quasi-local formalism. Following this approach we have to choose appropriate reference background. The most natural reference background is the Melvin space-time. With this choice following the detailed calculations presented in [17] we find that the mass of the static black Saturn is given by

$$
M=\frac{3 \pi}{8} \mathcal{R}^{2}\left[\zeta+\frac{2 \nu-1+\omega}{1+\omega}\right] .
$$




\subsection{Limits of the solution}

The solution has two natural non-singular limits. By removing the black hole from the configuration (i.e. by setting $\zeta=1$ ) we obtain the magnetized static black ring [16]. Removing the dipole black ring from the configuration we obtain the magnetized black hole. This is achieved by setting first $\omega=0$ and then taking the limit $\nu \rightarrow 0$.

\section{Discussion}

In this work we have constructed a new solution of the 5D EM equations describing a static black hole surrounded by a static dipole black ring. This Saturn-like configuration is balanced by the force yielded by the interaction of the dipole charge with an external self-gravitating magnetic field. The balanced solution depends on four independent parameters. Only one of them, the mass $M$, is a conserved quantity. Therefore our solution exhibits 3-fold continuous non-uniqueness. If we consider the solution in fixed Melvin background (i.e. keeping $B$ fixed) which is the most interesting case from physical point of view, the solution then exhibits 2-fold continuous non-uniqueness. Therefore our solution demonstrates 2 -fold continuous non-uniqueness of the 5D static, magnetized (neutral) black objects in fixed Melvin background. Let us denote by $\mathcal{A}^{\text {Saturn }}$ the total area of the magnetized static black Saturn and by $\mathcal{A}^{\text {hole }}$ and $\mathcal{A}^{\text {ring }}$ the area of the single magnetized static black hole and black ring. It can be shown that

$$
0<\mathcal{A}^{\text {ring }}<\mathcal{A}^{\text {Saturn }}<\mathcal{A}^{\text {hole }}
$$

for black configurations with the same total mass and immersed in the same Melvin background. Moreover, the magnetized static black Saturns swept the entire interval $\left(0, \mathcal{A}^{\text {hole }}\right)$.

\section{Acknowledgements}

The author would like to thank the Alexander von Humboldt Foundation for a stipend, and the Institut für Theoretische Physik Göttingen for its kind hospitality. The partial support by the Bulgarian National Science Fund under Grant MUF04/05 (MU 408) and VUF-201/06 is also acknowledged.

\section{References}

[1] R. Emparan and H. Reall, Phys. Rev. Lett. 88, 101101 (2002); hep-th/0110260

[2] R. Emparan, JHEP 0403, 064 (2004); hep-th/0402149

[3] H. Elvang, R. Emparan, D. Mateos and H. Reall, Phys. Rev. Lett. 93, 211302 (2004); hep-th/0407065

[4] A. Pomeransky and A. Sen'kov, Black ring with two angular momenta ; hep-th/0612005 
[5] S. Tomizawa, H. Ishihara, M. Kimura and K. Matsuno, Class. Quant. Grav. 24, 5609 (2007); arXiv:0705.1098[hep-th]

[6] J. Gauntlett and J. Gutowski, Phys. Rev. D71, 025013 (2005); hep-th/0408010

[7] J. Gauntlett and J. Gutowski, Phys. Rev. D71, 045002 (2005); hep-th/0408122

[8] H. Iguchi and T. Mishima, Phys. Rev. D75, 064018 (2007); hep-th/0701043

[9] H. Elvang and P. Figueras, JHEP 0705, 050 (2007); hep-th/0701035

[10] S. Yazadjiev, Phys. Rev. D76, 064011 (2007); arXiv:0705.1840[hep-th]

[11] J. Evslin and C. Krishnan, The Black Di-Ring: An Inverse Scattering Construction, arXiv:0706.1231[hep-th]

[12] K. Izumi, Orthogonal black di-ring solution, arXiv:0712.0902[hep-th]

[13] H. Elvang and M. Rodriguez,Bicycling Black Rings, arXiv:0712.2425[hep-th]

[14] S. Hollands and S. Yazadjiev, Uniqueness theorem for 5-dimensional black holes with two axial Killing fields, arXiv:0707.2775[gr-qc]

[15] S. Hollands and S. Yazadjiev, A Uniqueness theorem for 5-dimensional EinsteinMaxwell black holes, arXiv:0711.1722[gr-qc]

[16] M. Ortaggio, JHEP 05, 048 (2005); gr-qc/0410048

[17] S. Yazadjiev, Phys. Rev. D73, 064008 (2006); gr-qc/0511114

[18] S. Yazadjiev, Phys. Rev. D73, 104007 (2006); hep-th/0602116

[19] E. Radu, Mod. Phys. Lett. A17, 2277 (2002); gr-qc/0211035 